\title{
Proximal humerus reconstruction in orthopedic oncology
}

\author{
Sridhar Pinnamaneni ${ }^{1}$, Timothy A. Damron ${ }^{2}$ \\ 'Shoulder and Elbow Section, Signature Orthopedics, St. Louis, Missouri 63128, USA. \\ ${ }^{2}$ Orthopedic Oncology Section, Upstate Orthopedics, Syracuse, New York 13210, USA.
}

Correspondence to: Prof. Timothy A. Damron, Orthopedic Oncology Section, Upstate Orthopedics, Syracuse, New York 13210, USA.E-mail: damront@upstate.edu

How to cite this article: Pinnamaneni S, Damron TA. Proximal humerus reconstruction in orthopedic oncology. J Cancer Metastasis Treat 2021;7:7. http://dx.doi.org/10.20517/2394-4722.2020.94

Received: 2 Sep 2020 First Decision: 15 Oct 2020 Revised: 26 Oct 2020 Accepted: 24 Nov 2020 Published: 3 Feb 2021

Academic Editor: Ian Judson Copy Editor: Whitney Xu Production Editor: Jing Yu

\begin{abstract}
Proximal humeral reconstructive options following radical resection of proximal humeral primary and metastatic bone malignancies have evolved over time. With the relatively recent advent of the reverse total shoulder (RTSA), this technique has been increasingly employed in this setting over hemiarthroplasty techniques. An array of options, including proximal humeral allograft-prosthetic composites (including both RTSA and hemiarthroplasty), megaprostheses, and osteoarticular allografts, is reviewed from the perspective of their indications, techniques, complications, and published results. An extensive case-based pictorial presentation illustrates these options.
\end{abstract}

Keywords: Proximal humerus, oncology, primary bone tumor, metastatic disease, endoprosthesis, allograft

\section{INTRODUCTION}

The proximal humerus is a common site for metastatic and primary bone tumors ${ }^{[1,2]}$. With the evolution of chemotherapeutics, radiotherapy, and advanced imaging techniques, limb salvage surgery has become the norm in this location except for very advanced cases. Tumors in this region impose significant challenges for local control, reconstruction, and function. Potential glenohumeral joint involvement and close proximity of neurovascular structures affects local control and function, while lack of intrinsic stability and dependence on dynamic and static stabilizers affects reconstruction and function. Resection for metastatic disease may require bone-only resection, but restoration of soft-tissue attachments and glenohumeral

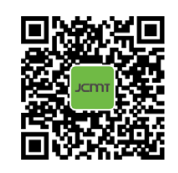


Table 1. Malawer surgical classification system of limb-sparing resections of the shoulder girdle

\begin{tabular}{ll}
\hline Type I & Intra-articular proximal humeral resection \\
Type II & Partial scapulectomy \\
Type III & Intra-articular total scapulectomy \\
Type IV & Extra-articular scapular and humeral head resection \\
Type V & Extra-articular humeral and glenoid resection \\
Type VI & Extra-articular humeral and total scapular resection \\
\hline
\end{tabular}

Table 2. Musculoskeletal tumor society functional evaluation (upper limb)

\begin{tabular}{|c|c|c|c|c|c|c|}
\hline Score & Pain & Function & $\begin{array}{c}\text { Emotional } \\
\text { acceptance }\end{array}$ & Hand positioning & Manual dexterity & Lifting ability \\
\hline 5 & No pain & No restriction & Enthused & Unlimited & No limitations & Normal load \\
\hline 4 & (Intermediate) & (Intermediate) & (Intermediate) & (Intermediate) & (Intermediate) & (Intermediate) \\
\hline 3 & Modest/non-disabling & $\begin{array}{l}\text { Recreational } \\
\text { restriction }\end{array}$ & Satisfied & Not above shoulder & $\begin{array}{l}\text { Loss of fine } \\
\text { movements }\end{array}$ & Limited (minor load) \\
\hline 2 & (Intermediate) & (Intermediate) & (Intermediate) & (Intermediate) & (Intermediate) & (Intermediate) \\
\hline 1 & $\begin{array}{l}\text { Moderate/ } \\
\text { intermittently disabling }\end{array}$ & $\begin{array}{l}\text { Partial occupational } \\
\text { restriction }\end{array}$ & Accepts & Not above waist & Cannot pinch & $\begin{array}{l}\text { Helping only(cannot } \\
\text { overcome gravity) }\end{array}$ \\
\hline 0 & $\begin{array}{l}\text { Severe/continuously } \\
\text { disabling }\end{array}$ & $\begin{array}{l}\text { Total occupational } \\
\text { restriction }\end{array}$ & Dislikes & None & Cannot grasp & Cannot move \\
\hline
\end{tabular}

Table 3. Henderson et al. ${ }^{[5]}$ classification of complications

\begin{tabular}{ll}
\hline Type of failure & \multicolumn{1}{c}{ Definition } \\
\hline 1 & Soft tissue failure \\
2 & Aseptic loosening \\
3 & Structural failure \\
4 & Infection \\
5 & Tumor progression \\
\hline
\end{tabular}

stability are challenging. Resection for primary bone tumors may require complete or partial resection of the deltoid, rotator cuff, joint capsule, axillary nerve, and portions of the scapula to achieve oncologic margins. Reconstruction after the latter resection creates even more complex reconstructive issues. The purpose of our article is to provide a comprehensive review of current reconstruction options after proximal humeral resection for oncologic reasons. This review is supplemented with case-based examples.

\section{CLASSIFICATION AND OUTCOME ASSESSMENT}

Classification of shoulder girdle resections according to Malawer range from types I to $\mathrm{VI}^{[3]}$. This classification is based on surgical margins, relationship of the tumor to other anatomic compartments, status of the glenohumeral joint, magnitude of the surgical procedures, and status of the abductor mechanism (deltoid/rotator cuff) [Table 1]. Functional and quality of life outcomes after treatment of musculoskeletal tumors are most commonly assessed with the Musculoskeletal Tumor Society (MSTS) score $^{[4]}$. This scale is based on questions about functional outcomes, pain, and emotional status [Table 2]. While it has been shown to be a reliable tool for the upper extremity, the MSTS score can overestimate function as compared to the patient-perceived score ${ }^{[4]}$. As for other sites, complications following these procedures are classified as described by Henderson et al. ${ }^{[5]}[$ Table 3].

\section{PREOPERATIVE CONSIDERATIONS}

In general, the orthopedic oncology patient has several specific characteristics that affect the choice of surgical reconstruction. The potential for a shortened survival, particularly in patients with diffuse metastatic disease, myeloma, and some primary bone tumors, should be considered. Orthopedic oncology patients often need a surgical solution that allows for immediate stability to allow restoration of function, 
especially in patients with poorer prognosis. Concomitant effects of radiotherapy and chemotherapy, including increased risk for scarring, wound healing problems, and infection, should also be considered. These factors differ from a non-oncology orthopedic patient and should be considered prior to finalizing a surgical plan.

The glenohumeral joint significantly relies on the dynamic stabilizers and force-couplers to allow for range of motion of the shoulder, including the rotator cuff, glenohumeral ligaments, and deltoid ${ }^{[6]}$. These structures comprise the functional anatomic compartment of the shoulder ${ }^{[7]}$. During wide resection of the proximal humerus for oncology surgery, major portions of the functional anatomic compartment are often removed. Those portions can specifically include all or the deep portion of the deltoid, subscapularis muscle belly, superior-posterior rotator cuff musculature, latissimus dorsi, brachialis, and portions of the triceps. Wide resection of this functional compartment during surgery creates a complex situation during reconstruction, especially when trying to restore both stability and function to the shoulder and proximal humerus ${ }^{[7]}$. When the rotator cuff is going to be compromised or resected, a reverse total shoulder arthroplasty (RTSA) implant should be considered. Need for resection of the brachial artery and/or all of the three major nerves affecting distal function (radial, median, and ulnar) are considered contraindications for limb salvage surgery, but resection of the axillary nerve is not.

\section{INDICATIONS}

The proximal humerus is a common location for upper extremity tumors. Common tumors requiring resection affecting the proximal humerus include metastatic bone disease, myeloma, primary bone sarcomas, and locally aggressive benign tumors.

\section{Metastatic Bone Disease/Myeloma}

Metastatic bone disease and myeloma commonly affect the proximal humerus. Common primary carcinomas that metastasize to bone include prostate, breast, kidney, thyroid, and lung. Breast and lung are the most common in females. Prostate and lung are the most common in males. Myeloma represents the most common primary bone cancer. In the setting of metastatic disease or myeloma involving the proximal humerus, there are limited indications for resection. Indications in these patients include: (1) solitary or oligometastatic renal cell carcinoma [Figures 1 and 2]; (2) extensive proximal humeral bone destruction that makes prophylactic internal fixation unreliable or simply not feasible [Figure 3]; (3) failure of other constructs due to tumor progression; and (4) hardware failure.

\section{Primary Bone Tumors}

The proximal humerus is a common location for primary sarcomas, including osteosarcoma, Ewing sarcoma, and chondrosarcoma ${ }^{[8]}$.

Osteosarcoma is the most common bone sarcoma overall and most common in pediatric patients ${ }^{[8]}$. The proximal humerus is the third most common location for osteosarcoma. The standard of care for treatment of osteosarcoma includes wide surgical resection with concomitant chemotherapy [Figure 4]. The typical metaphyseal location lends itself to intra-articular wide resection.

Ewing sarcoma is the second most common pediatric sarcoma ${ }^{[8]}$. Although Ewing sarcoma more commonly involves the diaphysis of long bones, it sometimes involves the metaphysis. The most common locations are the femur and tibia, but it can occur in the humerus. Treatment with surgical resection is an alternative to radiotherapy but favored whenever resected bone can be reconstructed, as it can in the proximal humerus.

Chondrosarcoma is the most common adult bone sarcoma ${ }^{[8]}$. Chondrosarcoma most frequently occurs in the pelvis, proximal femur, and proximal humerus. Treatment of grade 2 and 3 tumors is wide surgical 

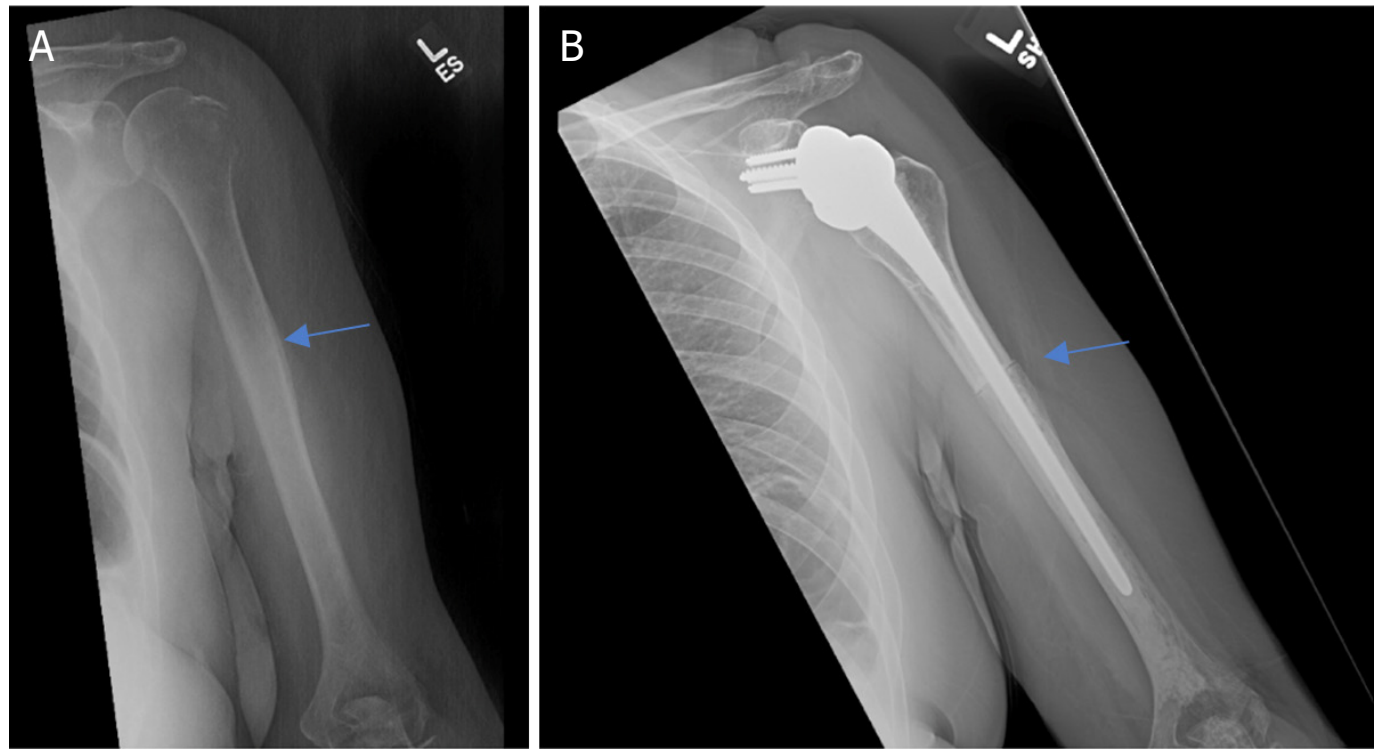

Figure 1. Anteroposterior radiographs of a 50 -year-old female with: a solitary renal cell carcinoma tumor of the proximal humerus preoperatively (A); one year after wide excision and proximal humeral APC reconstruction (B). Blue arrow notes the junction between the Allograft and the native bone
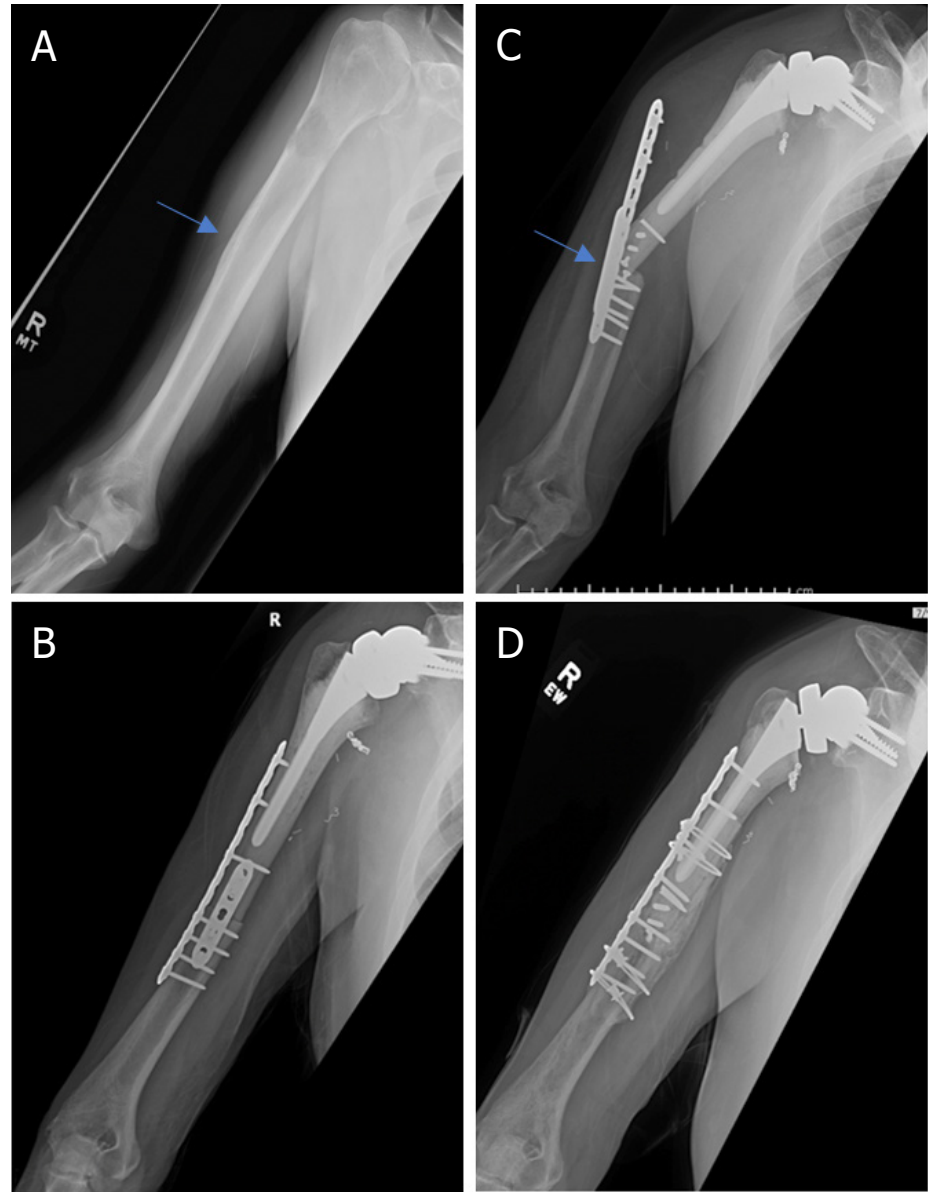

Figure 2. Anteroposterior radiographs of a 76-year-old male with: a solitary renal cell carcinoma tumor of the proximal humerus preoperatively (A); six months after wide excision and proximal humeral allograft prosthetic composite (APC) reconstruction with RTSA (B); one year postoperatively, patient represented with allograft-host junctional nonunion with hardware failure (C); anteroposterior radiographs after bone grafting, repair of the non-union with open reduction, and internal fixation (D) Blue arrow notes the junction between the Allograft and the native bone. 

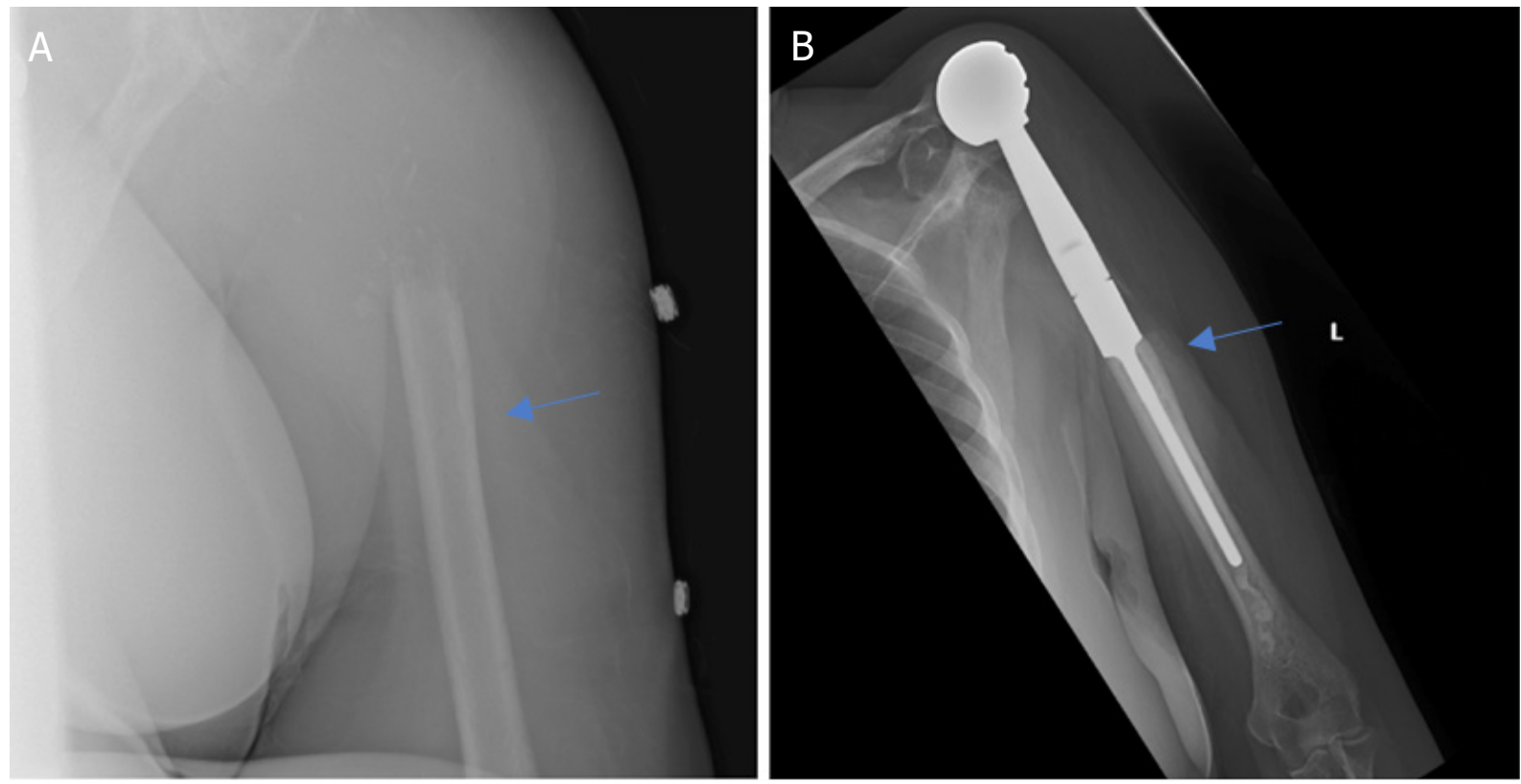

Figure 3. Anteroposterior radiographs of a 61-year-old female with: multiple myeloma of the proximal humerus preoperatively (A); five years after wide excision and proximal endoprosthesis reconstruction (B). Blue arrow notes the junction between the prosthesis and native bone.

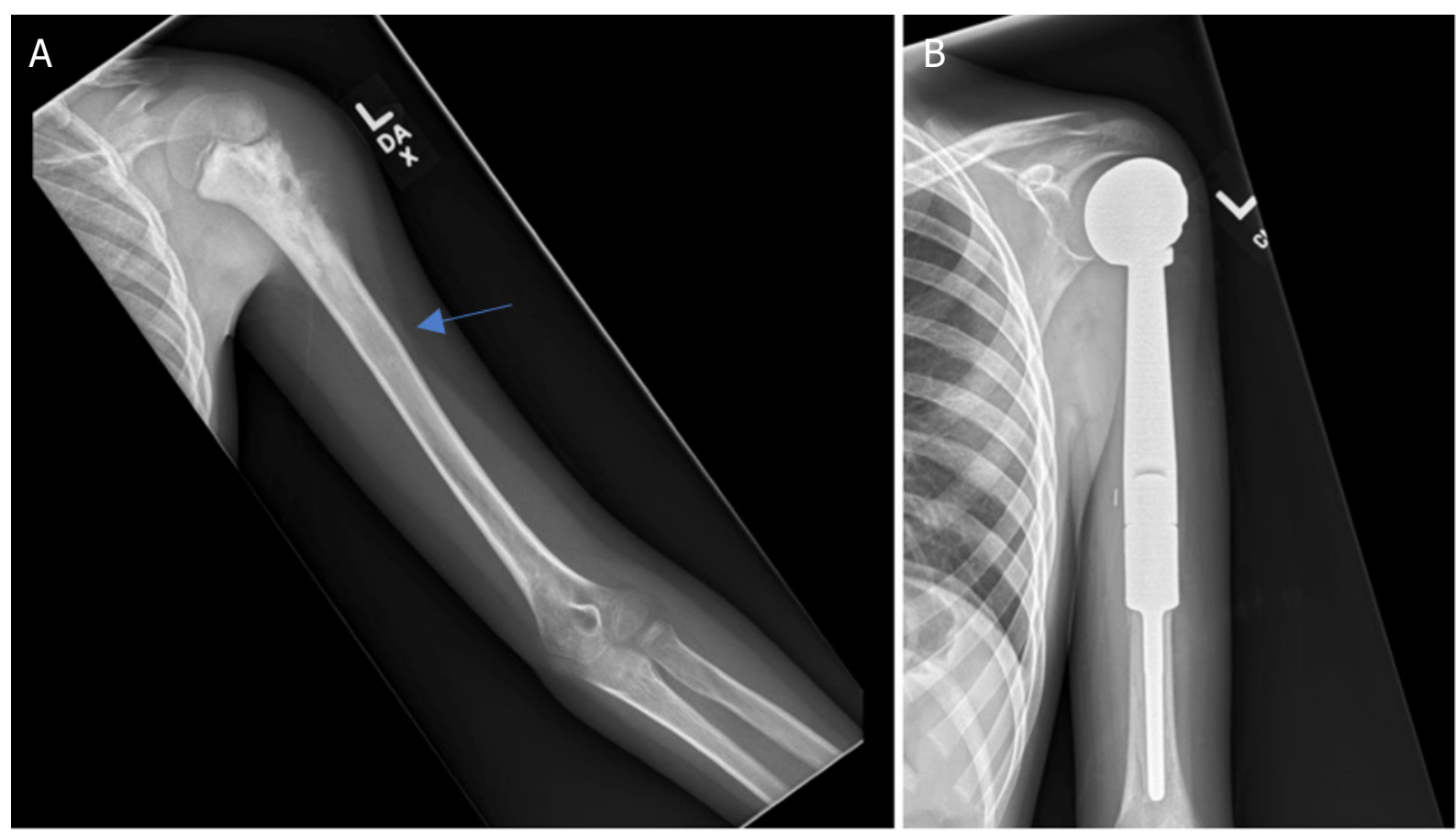

Figure 4. Anteroposterior radiographs of a 10-year-old male with: osteosarcoma of the proximal humerus preoperatively (A); after wide excision and proximal endoprosthesis reconstruction (B) Blue arrow notes the amount of resected bone 

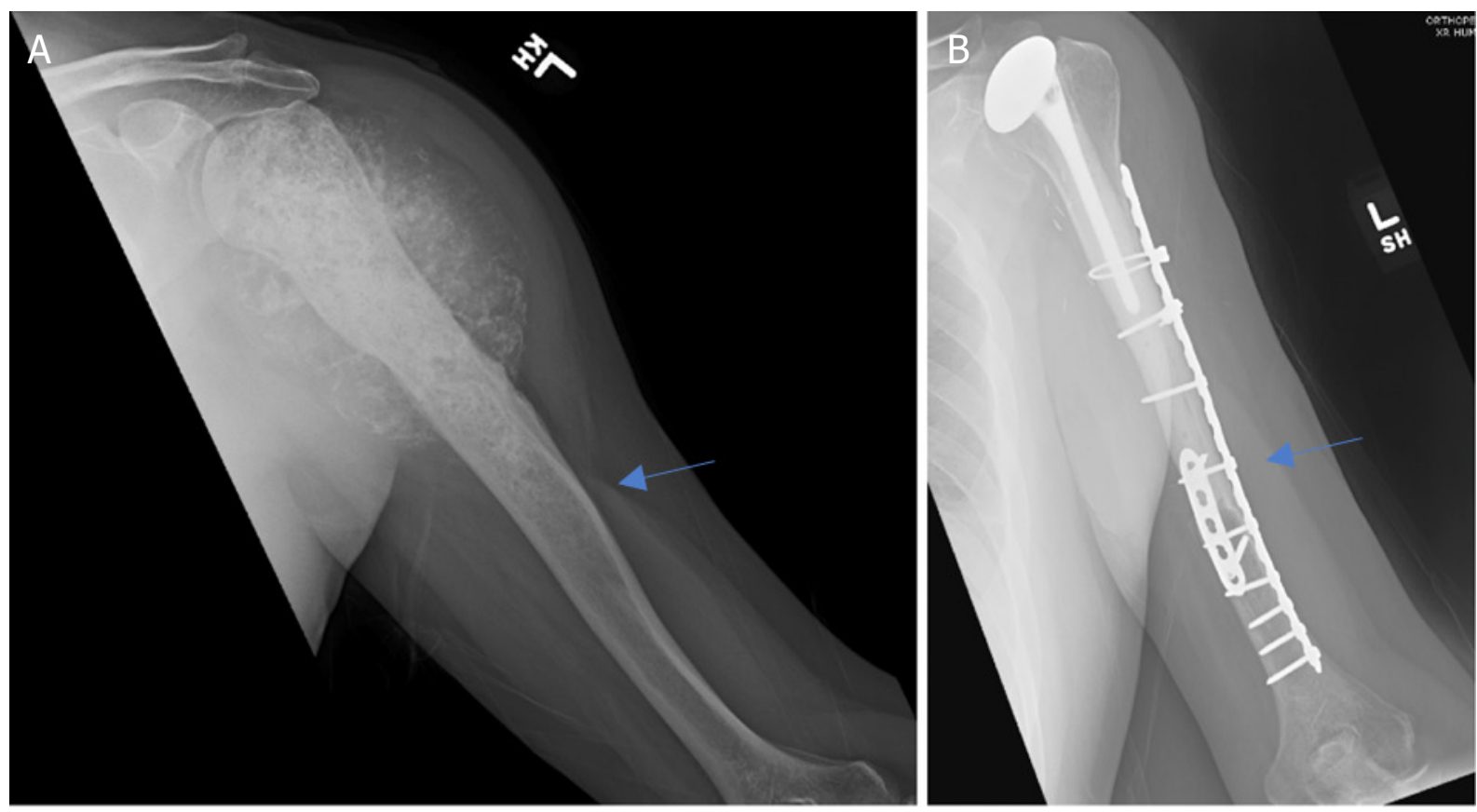

Figure 5. Anteroposterior radiographs of a 57-year-old male with: chondrosarcoma of the proximal humerus preoperatively (A); three years after wide excision and proximal humeral APC reconstruction (B). Blue arrow notes the junction between the allograft and native bone.

resection [Figures 5-7]. As for osteosarcoma, the typical metaphyseal location lends itself to intra-articular resection.

\section{Benign locally aggressive tumors}

Aggressive benign tumors with extensive bone destruction may necessitate resection if in the proximal humerus. While these indications are rare, giant cell tumor of the bone is the most likely benign tumor to require resection [Figure 8]. Rarely, aneurysmal bone cyst, osteoblastoma, and chondromyxoid fibroma require resection. Aneurysmal bone cyst is unlikely to require resection and reconstruction in pediatric patients, but they are particularly aggressive, secondary, or recurrent in an adult patient. Osteoblastoma is rare in the proximal humerus and necessitates resection only when it behaves aggressively, such as when it is hard to differentiate from osteosarcoma. Chondromyxoid fibroma is also an uncommon proximal humeral tumor but has the potential to behave aggressively.

\section{OPERATIVE OPTIONS}

\section{Osteoarticular allograft}

Reconstruction of the proximal humerus using an osteoarticular allograft [Figure 9] is a completely biologic articular reconstruction option.

Indications: Osteoarticular allograft reconstruction can be considered in pediatric and young patients due to concerns with prosthesis-related complications ${ }^{[9]}$. Contraindications include evidence of intra-articular tumor, inadequate host tissue to reconstruct the glenohumeral joint, and pre-existing glenohumeral arthrosis. A relative contraindication is need for radiotherapy, which may lead to non-union at the grafthost interface.

Technique: Although tissue matching is not required, size matching is important. The planned resection of the humerus should be measured on advanced imaging. This is important to ensure appropriate length 

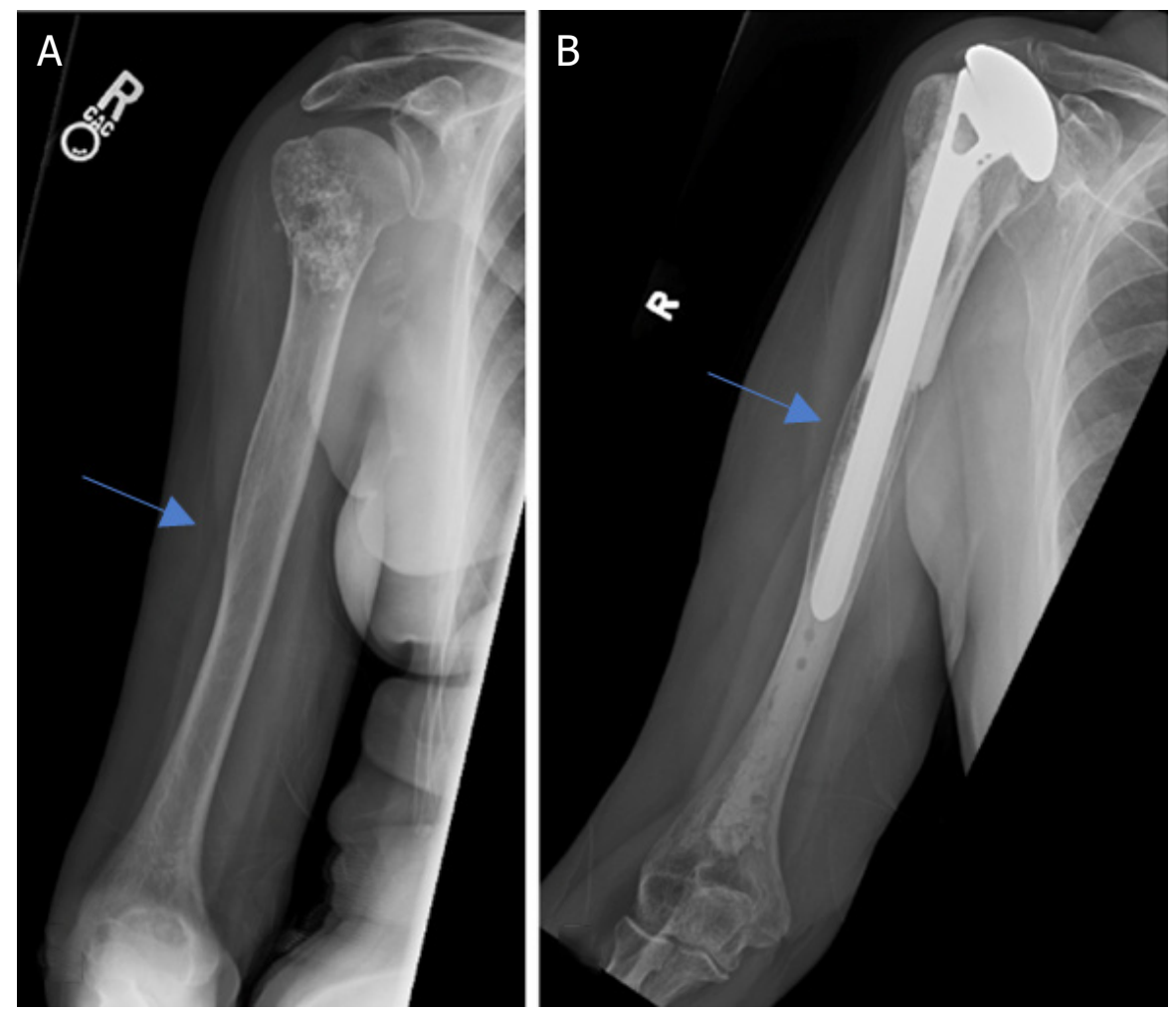

Figure 6. Anteroposterior radiographs of a 65-year-old male with: chondrosarcoma of the proximal humerus preoperatively (A); 4.5 years after wide excision and proximal humeral APC reconstruction (B). Blue arrow notes the junction between the allograft and native bone.

allograft is ordered and available. To preserve mechanical strength, non-irradiated bone should be used. To facilitate soft-tissue reconstruction, an allograft with soft-tissue attachments should be used. During surgery, the allograft can be taken out of the packaging and placed directly in a warm normal saline solution [Figure 9]. During the resection of the tumor, care should be taken to preserve the uninvolved glenohumeral capsule and tendon stumps of all the muscles resected, when possible. The glenohumeral capsule and the respective tendon stumps should be tagged with sutures for later identification. The humeral osteotomy should be completed in a transverse or step-wise fashion. The length of the resected native humerus should be measured.

The allograft should be tailored and cut to fit the native humeral osteotomy. It is important to have adequate length of the allograft humeral height to maintain adequate tension of the retained and repaired soft-tissue structures. The prepared allograft is placed into the proximal humeral defect, aligned, and any modification of the distal cut of the allograft should be performed. Next, the posterior, inferior, and anterior glenohumeral native capsule should be repaired to the allograft capsule with non-absorbable suture. The host rotator cuff can be repaired to the allograft rotator cuff attachments. This should provide enough stability of the glenohumeral joint to reduce the distal end of the allograft bone to the host humeral osteotomy site. Anatomic reduction of the host and donor bone is critical. A lateral locking compression plate is used for fixation. This fixation can be supplemented with an additional anterior shorter plate at 90 degrees to the lateral plate for additional biomechanical strength of the construct. Holes in the allograft should be minimized to avoid increased fracture risk but adequate for rigid fixation. Remaining transected tendons can be attached to the allograft soft-tissue attachments, particularly the latissimus dorsi, teres major, pec major, and deltoid ${ }^{[9]}$. Postoperatively, the patient is placed in a sling. Initially, the patient is nonweightbearing, and range of motion to the shoulder is limited. Once the soft-tissue healing is noted to be adequate, pendulum exercises and active assisted range of motion exercises can be initiated. 

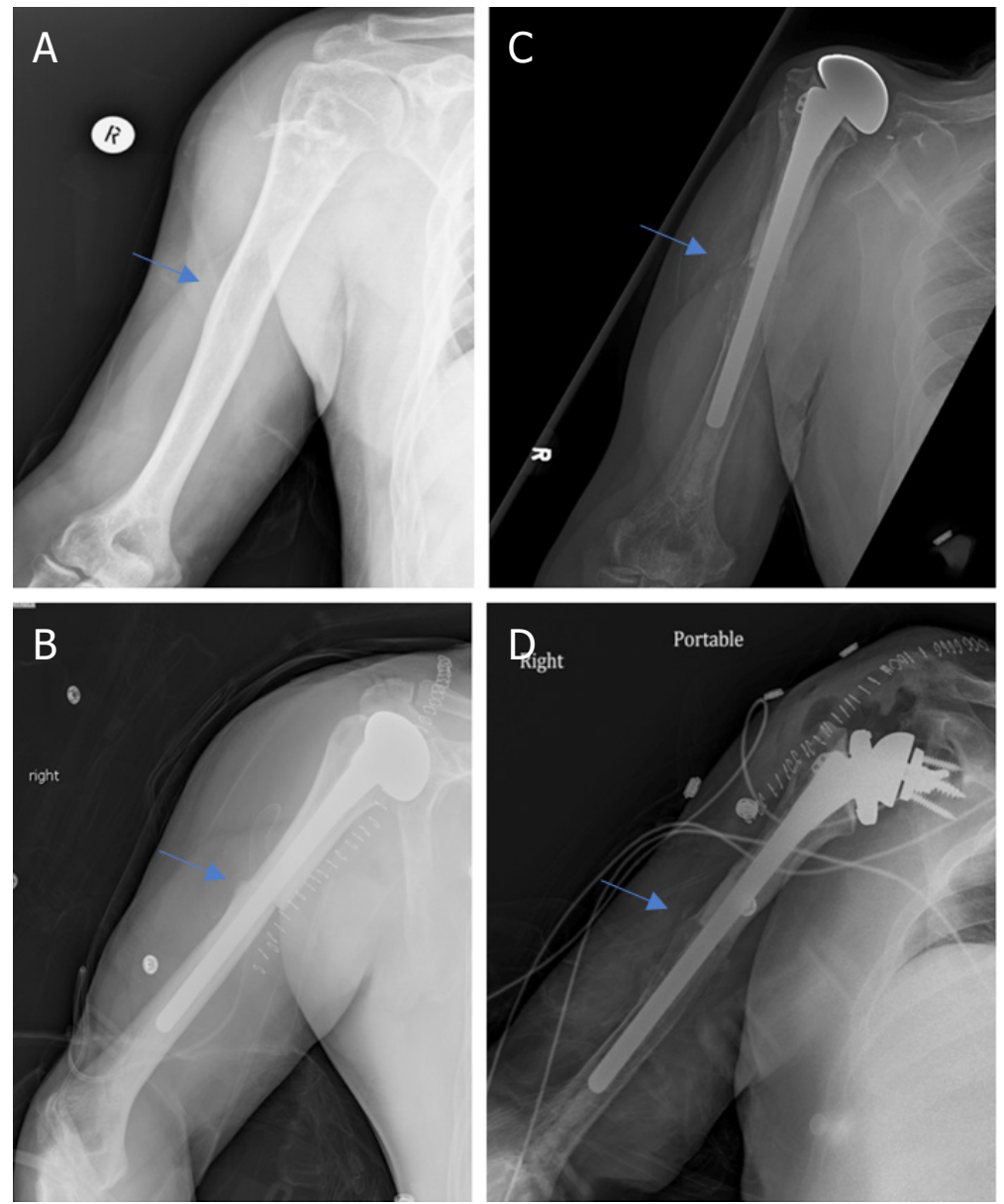

Figure 7. Anteroposterior radiographs of a 57-year-old male with: chondrosarcoma of the proximal humerus preoperatively (A); two months after wide excision and proximal humeral APC reconstruction with hemiarthroplasty (B); Seven years postoperatively, patient represented with excellent integration of the allograft but with proximal migration of the humeral head had worsening anterior superior escape on physical exam (C); anteroposterior radiographs immediately postoperatively after conversion from hemiarthroplasty to RTSA with glenosphere placement and modular exchange of the humeral head component for a humeral tray (D). Blue arrow notes the junction between the allograft and native bone.
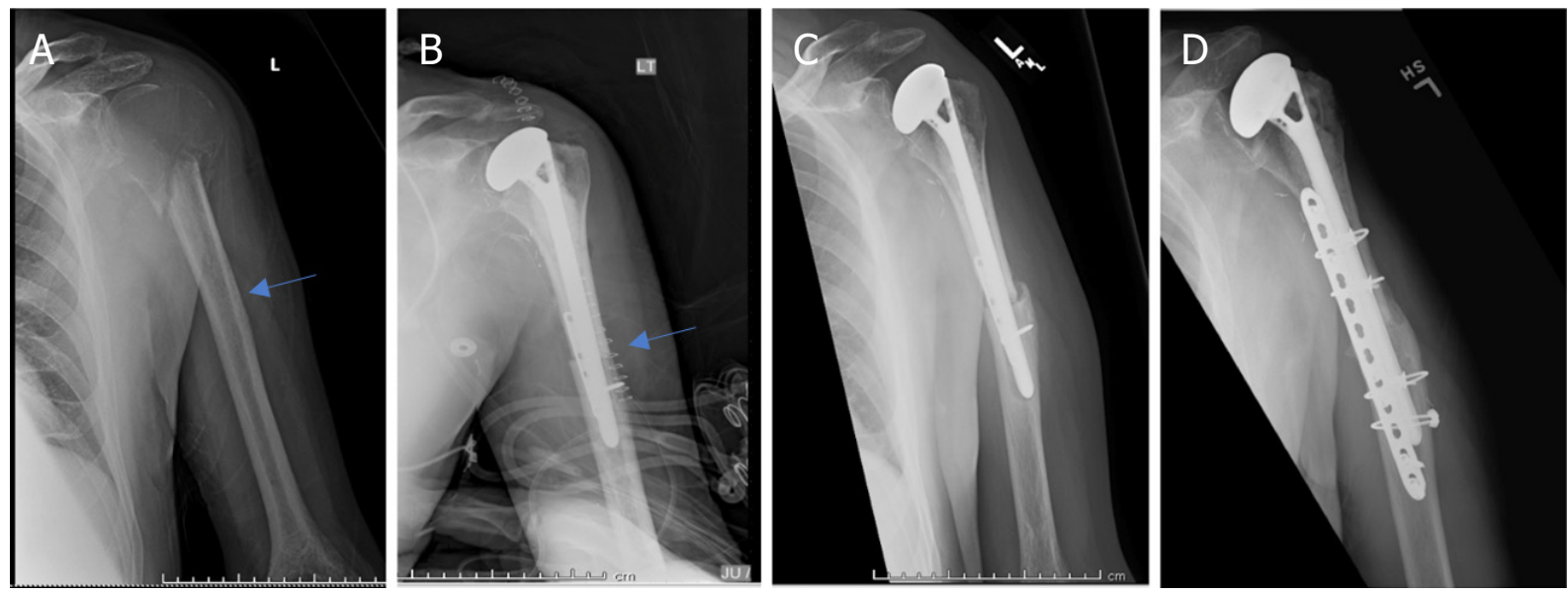

Figure 8. Anteroposterior radiographs of a 55-year-old male with: giant cell tumor of the proximal humerus preoperatively (A); six months after wide excision and proximal humeral APC reconstruction (B); patient was noted to have an allograft-host non-union with hardware failure at the two-year follow-up visit (C); anteroposterior radiographs after bone grafting, repair of the non-union with open reduction and internal fixation (D) Blue arrow notes that junction between allograft and native bone. 


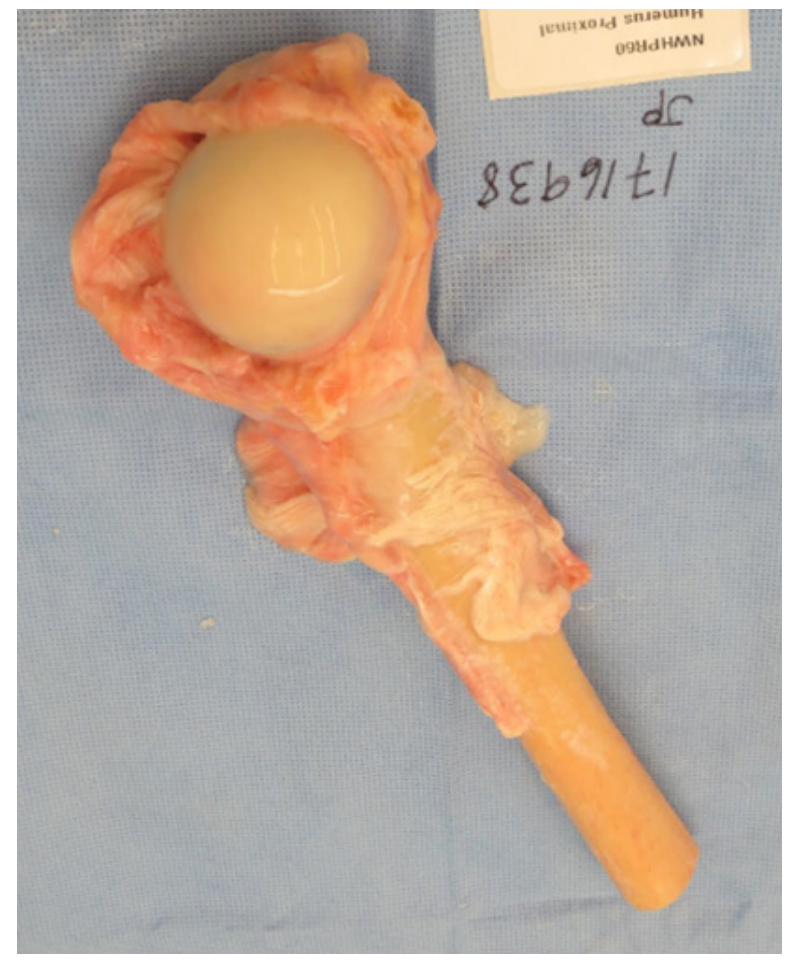

Figure 9. An example of a fresh frozen proximal humeral osteochondral allograft with preserved soft-tissue attachments. This allograft can be used in osteochondral allograft and allograft composite prosthesis reconstruction techniques

Table 4. Advantages and disadvantages of osteochondral allograft reconstruction

\begin{tabular}{ll}
\hline ADVANTAGES & DISADVANTAGES \\
\hline All-biologic reconstruction & Allograft osteolysis \\
Host-graft soft tissue attachments & Non-union \\
Continued boney and soft-tissue integration & Hardware failure \\
& Allograft fracture \\
& Allograft resorption \\
& Subchondral collapse \\
& Glenohumeral arthrosis \\
& Availability of allograft \\
& Poor long-term survivability \\
\hline
\end{tabular}

Advantages [Table 4]: The advantages of an osteoarticular allograft reconstruction include anatomic restoration of bone stock, glenohumeral joint capsule, rotator cuff, and surrounding dynamic shoulder stabilizers ${ }^{[10]}$. Presence of the soft-tissue attachments on an osteoarticular allograft facilitates host-to-graft soft-tissue repair ${ }^{[11]}$. Over time, host-to-graft healing can occur with the host tissue healing to the softtissue bone components of the allograft, unlike with a metallic endoprosthesis.

Disadvantages [Table 4]: Reported complication rates have been relatively high ${ }^{[9,10]}$. Allograft specific complications include allograft osteolysis, non-union, hardware failure, resorption, subchondral collapse, glenohumeral arthrosis, and allograft fracture. These specific complications are related to the use of allograft and the potential problems with using allograft tissue for reconstruction. Ubiquitous potential problems with resection/reconstruction of the proximal humerus include glenohumeral subluxation or dislocation, infection, wound healing problems, and local recurrence. Additionally, development of glenohumeral arthrosis can occur after osteochondral allograft reconstruction and result in continued pain and dysfunction ${ }^{[12]}$. In addition to complications, appropriately sized and procured osteoarticular allografts 
may be difficult to locate depending on access to tissue banks and available stock. The treatment of these potential complications is case dependent.

Outcomes: A 1990 series of 16 patients from Massachusetts General reported 68\% survivorship at 5 years ${ }^{[13]}$. Mean MSTS score decreased from $81 \%$ at 14 months to $70 \%$ at 34 months ${ }^{[13]}$. In a 2015 series of 19 patients from the Buenos Aires, graft survival rate was $55 \%$ at 10 years with mean MSTS score of $76 \%$ in patients with retained allografts ${ }^{[9]}$.

\section{Endoprosthesis}

Reconstruction of the proximal humerus may also be done with an endoprosthesis construct using readily available modular implants [Figures 3 and 4]. Their modularity allows for restoration of humeral height, soft-tissue tensioning, and amount of constraint but does not provide the potential for soft-tissue healing to the reconstruction.

Indications: Endoprosthesis reconstructions should be considered in patients who have intra-articular tumor involvement and those with limited expected survival who need immediate stability and early functionality. Most endoprosthesis implants are usually implanted as hemiarthroplasties [Figures 3 and 4], but some modern endoprosthesis implants have the convertibility to be placed in a reverse total shoulder arthroplasty (RTSA) configuration and accommodate a glenosphere.

Technique: As for any planned proximal humeral resection/reconstruction, the planned length of resection should be measured on preoperative advanced imaging, and preservation of soft tissues that can be repaired during the reconstruction should be accomplished during the resection. The deltoid insertion should be spared if possible. The glenohumeral capsule and the respective tendon stumps should be tagged with sutures for later identification. These structures are useful in achieving joint stability by attaching these soft-tissue attachments to the endoprosthesis. After measuring the resected specimen, the defect is reconstructed by combining the modular humeral head, body, and shaft endoprosthetic components to achieve optimal soft-tissue tensioning. If adequate distal fixation is not achieved with uncemented intramedullary stem, cemented techniques should be used.

Soft-tissue repair to endoprosthesis can utilize attachment of soft tissue proximally to holes in the endoprosthesis with non-absorbable sutures. Soft-tissue repair can be augmented by an aortic synthetic mesh graft placed over the endoprosthesis to which the soft tissues can be sutured ${ }^{[14]}$.

In RTSA endoprostheses, adequate lateralization and standard glenosphere placement techniques should be used. Since endoprosthetic reconstruction after oncologic resection has a higher instability risk compared to non-oncology patients, when using a RTSA, techniques to increase stability include increased lateralization of the glenosphere, a larger glenosphere, and a constrained humeral insert.

Postoperatively, the patient is placed in a sling. Initially, the patient is non-weightbearing and range of motion to the shoulder is limited. Often with endoprosthesis reconstruction, immediate light weightbearing and active assisted range of motion exercises can be initiated.

Advantages [Table 5]: The advantages of endoprosthesis reconstruction are modularity, early return to function, lower reported complication rates, and superior implant survival ${ }^{[15-17]}$. This reconstruction option also eliminates allograft specific concerns including allograft-host integration, subchondral collapse, allograft fractures, and non-union. 
Table 5. Advantages and disadvantages of endoprosthesis reconstruction

\begin{tabular}{ll}
\hline Advantages & \multicolumn{1}{c}{ Disadvantages } \\
\hline Modularity of prosthesis & Proximal Migration (Hemiarthroplasty) \\
Convertibility of prosthesis & Instability \\
Immediate stability & Aseptic Loosening \\
Early range of motion and functionality & Metal-soft tissue attachments \\
Lower complication rates compared to osteochondral allograft & Infection \\
No need for allograft & \\
Good long-term survivability & \\
Good long-term functional outcomes & \\
\hline
\end{tabular}

Disadvantages [Table 5]: The disadvantages of endoprosthesis reconstruction include increased risk for dislocation, proximal migration [Figure 3], and infection. Decreased capacity for restoration of softtissue attachments due to the non-biologic metal-soft-tissue interface contributes to the potential for instability ${ }^{[16,18]}$. Proximal migration [Figure 3], subluxations, and dislocations are common with this reconstruction ${ }^{[19-21]}$. Ross et al. ${ }^{[18]}$ reported proximal migration, subluxation or dislocations in 16/25 endoprosthetic proximal humeral reconstructions. Endoprosthetic complication rates between $4.5 \%$ and $85 \%$ have been reported, with the most common complications including proximal migration, subluxation, infection, and aseptic loosening ${ }^{[17]}$.

Outcomes: This reconstruction technique can result in good functional outcomes, as shown in a systemic review performed in $2014^{[17]}$. In this systemic review, MSTS functional scores ranged from $61 \%$ to $77 \%$ over 10 studies in 141 patients $^{[17]}$. Implant survival ranged from $38 \%$ to $100 \%$ over 17 studies in 341 patients ${ }^{[17]}$. In another case series of 39 patients, Raiss reported $72 \%$ survivorship with average three-year follow-up ${ }^{[22]}$.

\section{Allograft Prosthetic Composite}

Reconstruction of the proximal humerus using an allograft prosthetic composite (APC) provides a hybrid of allograft and prosthetic reconstruction techniques [Figure 5]. The APC was initially designed in hip and knee surgery to address the problems with resorption, fracture, and cartilage degeneration in osteochondral allograft reconstructions. This reconstruction option has a hybrid advantage and disadvantage profile combining some of the advantages and disadvantages of both the all biologic osteochondral allograft and the endoprosthesis reconstructive options.

Indications: In patients requiring substantial bone and soft-tissue resections, an APC can help decreased the risk of dislocations and instability associated with an endoprosthesis reconstruction. In situations where there is tumor involvement of the glenohumeral joint, an extra-articular resection is needed to limit the risk of local recurrence. If there is pre-existing glenohumeral arthrosis in conjunction with significant softtissue resection, an APC can be a viable reconstruction option. In situations where the rotator cuff has to be resected or compromised, but the deltoid function can be preserved, a RTSA can be used [Figures 1 and 2] $]^{[17]}$. The RTSA is more constrained, and therefore there is decreased risk of proximal migration and instability. The RTSA accomplishes this by moving the center of rotation more inferior and medial allowing the deltoid to act with a longer lever arm.

Technique: Prior to surgery, the planned resection of the humerus should be measured on the preoperative advanced imaging $^{[23]}$. This is important to ensure that the appropriate length proximal humeral allograft is available during the surgery. During the surgery, the allograft can be taken out of the packaging and placed directly in a warm normal saline solution [Figure 9]. During the resection of the tumor, care should be taken to preserve the uninvolved glenohumeral capsule and tendon stumps of all the muscles resected. The deltoid insertion should be spared if possible. The glenohumeral capsule and the respective tendon stumps should be tagged with sutures for later identification. Once the resection and the humeral osteotomy is 
completed, the humeral length of the resected native humerus should be measured. The osteotomy should be completed in either a transverse fashion or a step-wise fashion. Next, the humerus allograft should be prepped on the back table. The allograft should be tailored and cut to fit the native humeral osteotomy. It is important to have adequate length of the allograft humeral height to maintain adequate tension of the repaired soft-tissue structures. Next, the allograft should be prepped on the back table using the implant system of choice. The allograft should be prepped according to the appropriate broaches and reamers to accommodate the chosen implant. At this point, if a RTSA is being completed, the glenosphere should be placed. In the case of using a RTSA, adequate lateralization and standard glenosphere placement techniques should be used. Since these patients are at a higher risk for instability when compared to non-oncology patients, techniques to increases stability of the RTSA should be used including increased lateralization of the glenosphere, placement of a larger glenosphere, and using a constraint humeral insert. Next, the implant with the appropriate humeral stem implant should be implanted into the allograft. The implant should be long enough to incorporate approximately two cortical diameters distal to the native humeral osteotomy site. Next, the distal portion of the implant should be placed into the native humeral canal. Cement techniques should be used to fix the distal portion of the implant into the native humeral canal. Prior to final cementation, the humeral length and soft-tissue tensioning should be trailed using a trial prosthesis. Then, the distal portion of the implant is cemented into the native humeral bone. The decision to place a supplementary plate can be made at the discretion of the surgeon [Figure 5]. A locking compression plate can be used for fixation. This fixation can be supplemented with an additional anterior based short plate. Next, the posterior, inferior, and anterior glenohumeral native capsule should be repaired to the allograft capsule with non-absorbable suture. The host rotator cuff should be repaired to the allograft rotator cuff attachments. Next, the remaining resected muscles can be attached including the latissimus dorsi, teres major, pec major, deltoid, etc. Finally, the remaining superficial soft-tissue structures can be closed.

Postoperatively, the patient is placed in a sling. Initially, the patient is non-weightbearing and range of motion to the shoulder is limited. Active assisted shoulder range of motion can be initiated after the first week. Once healing of the allograft-host interface is noted to be adequate, weightbearing can progressively increased.

Advantages [Table 6]: The advantages of this type of reconstruction include anatomic restoration of the bone stock, glenohumeral joint, and surrounding soft tissues. This reconstruction option allows for hostto-graft soft-tissue attachments to attach host tendons, including the latissimus dorsi, deltoid, rotator cuff, etc ${ }^{[11]}$. Over time, the soft-tissue attachments and bony components of the allograft can be fully incorporated into the host bone and soft tissues over time, unlike with a prosthesis. Additionally, this reconstruction method can address any pre-existing glenohumeral arthrosis. Placement of standard hemiarthroplasty or RTSA implants can be used which allows for more modularity and lower cost. The patient also gets immediate stability and early functionality of the shoulder compared to an osteoarticular allograft.

Disadvantages [Table 6]: The disadvantages include allograft-related complications including allograft osteolysis, non-union [Figures 2 and 8], hardware failure, allograft fracture, allograft resorption, stress shielding, and delayed union ${ }^{[24]}$. Additionally, instability, aseptic loosening, and proximal migration [Figures 6 and 7] should be considered ${ }^{[25]}$. Availability of tissue banks and variety of allograft options to include soft-tissue attachments and enough resected bone stock can be variable depending on geographic location.

Outcomes: A case series of 36 patients treated with APC with an average follow-up of six years showed that the average MSTS score at the last follow-up was 26 out of 30 points with an $88 \%$ survivorship at 10 years ${ }^{[25]}$. Reconstruction with RTSA has shown good functional outcomes as well, with mean MSTS scores reported 
Table 6. Advantages and disadvantages of allograft prosthetic composite reconstruction

\begin{tabular}{ll}
\hline Advantages & Disadvantages \\
\hline All-biologic reconstruction & Allograft osteolysis \\
Host-Graft soft tissue attachments & Non-union \\
Modularity of prosthesis & Hardware failure \\
Convertibility of prosthesis & Allograft fracture \\
Immediate stability & Allograft resorption \\
Early range of motion and functionality & Proximal migration (Hemiarthroplasty) \\
Lower complication rates compared to osteochondral allograft & Instability \\
Can use standard implants instead of endoprosthesis implants & Aseptic loosening \\
Good long-term survivability & Availability of allograft \\
Good long-term functional outcomes & \\
\hline
\end{tabular}

from $60 \%$ and $79 \%$ in different case series ${ }^{[15,26-28]}$. In general, numerus studies have shown better MSTS scores and better abduction in patient with intact deltoid ${ }^{[24-27]}$.

\section{Other Surgical Options}

Other proximal humeral reconstruction options include the Clavicula pro humero and glenohumeral arthrodesis. Alternatively, a Tikhoff-Linberg procedure can be performed for salvage cases. The indications for these procedures are rare.

\section{CONCLUSION}

Limb salvage surgery with wide resection and reconstruction is considered the standard of care for a number of oncologic indications including primary bone tumors and metastatic disease. Wide resections of the proximal humerus and the surrounding soft-tissue stabilizing structures necessitate a complex reconstruction. While there are a number of case series reviewing different types of reconstruction options after proximal humeral resection for tumor, there are no high-level comparative studies in the literature. Considering this, there is little agreement on the ideal method, but reverse total shoulder reconstruction has been increasingly used.

\section{DECLARATIONS}

\section{Authors' contributions}

Completing composition of this article: Pinnamaneni S, Damron TA

Providing cases in this article: Damron TA

\section{Availability of data and materials}

Not applicable.

\section{Financial support and sponsorship}

Not applicable.

\section{Conflicts of interest}

Not applicable.

\section{Ethical approval and consent to participate}

Written informed consent to participate and consent for publication from the patients was obtained prior to surgical intervention.

\section{Consent for publication}

The consent for publication was obtained from patients included in the study. 


\section{Copyright}

(c) The Author(s) 2021.

\section{REFERENCES}

1. Hennessy DW, Raskin KA, Schwab JH, Lozano-Calderón SA. Endoprosthetic reconstruction of the upper extremity in oncologic surgery. J Am Acad Orthop Surg 2020;28:e319-27.

2. Pisters PW, Leung DH, Woodruff J, Shi W, Brennan MF. Analysis of prognostic factors in 1,041 patients with localized soft tissue sarcomas of the extremities. J Clin Oncol 1996;14:1679-89.

3. Malawer MM, Meller I, Dunham WK. A new surgical classification system for shoulder-girdle resections. Analysis of 38 patients. Clin Orthop Relat Res 1991;267:33-44.

4. Janssen SJ, van Rein EA, Paulino Pereira NR, et al. The discrepancy between patient and clinician reported function in extremity bone metastases. Sarcoma 2016;2016:1014248.

5. Henderson ER, Groundland JS, Pala E, et al. Failure mode classification for tumor endoprostheses: retrospective review of five institutions and a literature review. J Bone Joint Surg Am 2011;93:418-29.

6. Clark JM, Harryman DT. Tendons, ligaments, and capsule of the rotator cuff. Gross and microscopic anatomy. The Journal of Bone \& Joint Surgery 1992;74:713-25.

7. Malawer M, Wittig J. Overview of resections around the shoulder girdle: anatomy, surgical considerations and classification. Musculoskeletal Cancer Surgery 2006:179-202

8. Lee DH, Hills JM, Jordanov MI, Jaffe KA. Common tumors and tumor-like lesions of the shoulder. J Am Acad Orthop Surg 2019;27:236-45.

9. Farfalli GL, Ayerza MA, Muscolo DL, Aponte-Tinao LA. Proximal humeral osteoarticular allografts: technique, pearls and pitfalls, outcomes. Curr Rev Musculoskelet Med 2015;8:334-8.

10. Gebhardt MC, Roth YF, Mankin HJ. Osteoarticular allografts for reconstruction in the proximal part of the humerus after excision of a musculoskeletal tumor. J Bone Joint Surg Am 1990;72:334-45.

11. Wang Z, Guo Z, Li J, Li XD, Sang HX. Functional outcomes and complications of reconstruction of the proximal humerus after intraarticular tumor resection. Orthop Surg 2010;2:19-26.

12. Mankin HJ, Gebhardt MC, Jennings LC, Springfield DS, Tomford WW. Long-term results of allograft replacement in the management of bone tumors. Clin Orthop Relat Res 1996;324:86-97.

13. Getty PJ, Peabody TD. Complications and functional outcomes of reconstruction with an osteoarticular allograft after intra-articular resection of the proximal aspect of the humerus. J Bone Joint Surg Am 1999;81:1138-46.

14. Marulanda GA, Henderson E, Cheong D, Letson GD. Proximal and total humerus reconstruction with the use of an aortograft mesh. Clin Orthop Relat Res 2010;468:2896-903.

15. van de Sande MA, Dijkstra PD, Taminiau AH. Proximal humerus reconstruction after tumour resection: biological versus endoprosthetic reconstruction. Int Orthop 2011;35:1375-80.

16. Kumar D, Grimer RJ, Abudu A, Carter SR, Tillman RM. Endoprosthetic replacement of the proximal humerus. Long-term results. $J$ Bone Joint Surg Br 2003;85:717-22.

17. Teunis T, Nota SP, Hornicek FJ, Schwab JH, Lozano-Calderón SA. Outcome after reconstruction of the proximal humerus for tumor resection: a systematic review. Clin Orthop Relat Res 2014;472:2245-53.

18. Ross AC, Sneath RS, Scales JT. Endoprosthetic replacement of the humerus and elbow joint. J Bone Joint Surg Br 1987;69:652-5.

19. Cannon CP, Paraliticci GU, Lin PP, Lewis VO, Yasko AW. Functional outcome following endoprosthetic reconstruction of the proximal humerus. J Shoulder Elbow Surg 2009;18:705-10.

20. Scotti C, Camnasio F, Peretti GM, Fontana F, Fraschini G. Modular prostheses in the treatment of proximal humerus metastases: review of 40 cases. J Orthop Traumatol 2008;9:5-10.

21. Mayilvahanan N, Paraskumar M, Sivaseelam A, Natarajan S. Custom mega-prosthetic replacement for proximal humeral tumours. Int Orthop 2006;30:158-62.

22. Raiss P, Kinkel S, Sauter U, Bruckner T, Lehner B. Replacement of the proximal humerus with MUTARS tumor endoprostheses. Eur $J$ Surg Oncol 2010;36:371-7.

23. Lozano-Calderón SA, Chen N. Proximal humerus allograft prosthetic composites: technique, outcomes, and pearls and pitfalls. Curr Rev Musculoskelet Med 2015;8:324-33.

24. Ruggieri P, Mavrogenis AF, Guerra G, Mercuri M. Preliminary results after reconstruction of bony defects of the proximal humerus with an allograft-resurfacing composite. J Bone Joint Surg Br 2011;93:1098-103.

25. Abdeen A, Hoang BH, Athanasian EA, Morris CD, Boland PJ, Healey JH. Allograft-prosthesis composite reconstruction of the proximal part of the humerus: functional outcome and survivorship. J Bone Joint Surg Am 2009;91:2406-15.

26. Maclean S, Malik SS, Evans S, Gregory J, Jeys L. Reverse shoulder endoprosthesis for pathologic lesions of the proximal humerus: a minimum 3-year follow-up. J Shoulder Elbow Surg 2017;26:1990-4.

27. Griffiths D, Gikas PD, Jowett C, et al. Proximal humeral replacement using a fixed-fulcrum endoprosthesis. J Bone Joint Surg Br 2011;93:399-403.

28. Potter BK, Adams SC, Pitcher JD Jr, Malinin TI, Temple HT. Proximal humerus reconstructions for tumors. Clin Orthop Relat Res 2009;467:1035-41. 